\title{
Nanotriangle Decorated Silicon Nitride Waveguides for Integrated Surface-Enhanced Raman Spectroscopy
}

\author{
Pieter C. Wuytens ${ }^{1,2.3}$ Andre G. Skirtach ${ }^{2,3}$ and Roel Baets ${ }^{1,3}$ \\ ${ }^{I}$ Photonics Research Group, Department of Information Technology, Ghent University - imec, B-9052 Ghent, Belgium \\ ${ }^{2}$ Department of Molecular Biotechnology, Ghent University, B-9000 Ghent, Belgium \\ Center for Nano- and BioPhotonics, Ghent University, B-9000 Ghent, Belgium \\ pieter.wuytens@ugent.be
}

\begin{abstract}
Nanosphere lithography provides an e-beam free method for patterning gold nanoplasmonic antennas. By combining this technique with deep-UV photolithography, we fabricate $\mathrm{Si}_{3} \mathrm{~N}_{4}$ waveguides interfaced to plasmonic antennas capable of exciting and collecting surface-enhanced Raman spectra.

OCIS codes: (130.0130) Integrated optics; (130.6010) Sensors; (240.6695) Surface-enhanced Raman scattering; (220.4241) Nanostructure fabrication;
\end{abstract}

Surface-enhanced Raman spectroscopy (SERS) has become an established technique for a sensitive and selective detection in a myriad of applications[1]. Following the development of waveguide-based Raman spectroscopy[2-4], recent efforts successfully combined gold nano-antennas with dielectric photonic waveguides at visible wavelengths[5,6]. This led to first demonstrations of waveguide-based excitation and collection of surface-enhanced Raman scattering [6]. However, current approaches rely on very challenging fabrication schemes using (multiple) ebeam exposures with both critical alignment and resolution. Not only are these processes resource-intensive, they are also hard to combine with already patterned photonic circuits. As a consequence, the full potential of a mature integrated technology remains out of reach for SERS-based products. Important examples of these are the silicon or silicon nitride photonics platforms enabled by wafer-scale deep-UV patterning in a CMOS-fab [7]. In contrast, it is exactly this access to optimized and mass reproducible photonic components such as filters, modulators and spectrometers that drives continuing success of other types of integrated optical biosensors.

Here, we introduce a nanosphere-lithography based method for patterning gold nanotriangles on deep-UV patterned $\mathrm{Si}_{3} \mathrm{~N}_{4}$ photonic waveguides. We demonstrate excitation and collection of surface-enhanced Raman spectra using this waveguide. To the best of our knowledge, this is the first demonstration of a waveguide-based, e-beam free SERS-platform. It opens a route towards a complete on-chip Raman spectrometer combining both the sensing area, the spectrometer and even the pump source.

We start from a $4 \mathrm{~cm}^{2}$ deep-UV patterned dye containing $220 \mathrm{~nm}$ thick and $1.6 \mu \mathrm{m}$ wide $\mathrm{Si}_{3} \mathrm{~N}_{4}$ strip waveguides on a $2.4 \mu \mathrm{m} \mathrm{SiO}{ }_{2}$ layer on $\mathrm{Si}$. On top of this chip a $5 \mu \mathrm{m}$ wide opening is patterned in photoresist (AZ MiR 701) using UV contact lithography. After thinning down the resist to $400 \mathrm{~nm}$ using $\mathrm{O}_{2}$ plasma (Vision 320 RIE, 50 sccm $\mathrm{O}_{2}, 75 \mathrm{~W}, 100 \mathrm{mTorr}, 235 \mathrm{~s}$ ), polystyrene beads with a $448 \mathrm{~nm}$ diameter are spin coated at an optimized speed, dilution and acceleration in order to acquire a hexagonally packed monolayer of beads in the opening on top of the waveguide (fig 1(a)). Next, a short $\mathrm{O}_{2}$ plasma $(15 \mathrm{~s})$ is used to, on the one hand, ensure a good metal adhesion and, on the other hand, reduce the size of the beads such that final nanotriangles will have an optimal localized surface plasmon resonance for Raman excitation at $785 \mathrm{~nm}$. These beads subsequently act as a mask for evaporating a $2 \mathrm{~nm}$ Ti adhesion layer and a $70 \mathrm{~nm}$ Au layer. After gold deposition, the beads and photoresist are lifted off, revealing the periodic array of gold nanotriangles, as well as some larger structures as a consequence of packing defects in the
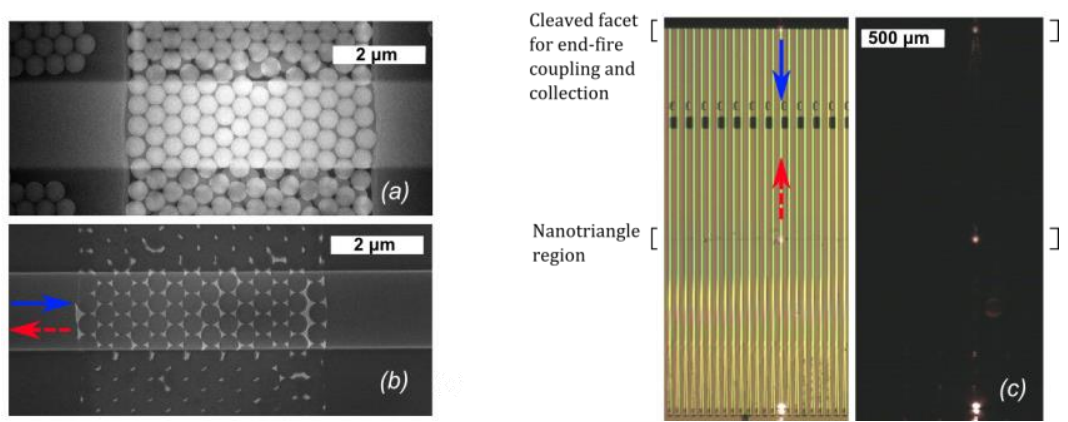

Figure -1 (a) SEM images showing a hexagonally packed monolayer of polystyrene beads in a stripe patterned on a SiN strip waveguide and (b) the resulting gold pattern after lifting of the photoresist and beads. The blue solid arrows indicate the propagation of the pump light along the waveguide, the red dashed arrow the direction of the collected Stokes scattered light. (c) Top-view of the chip when end-fire coupled showing an array of waveguides patterned with nanotriangles (left). The scattering of the 785 pump laser is clearly visible at the position of the entrance facet, nanotriangle region and grating coupler at the end of the waveguide (right). 

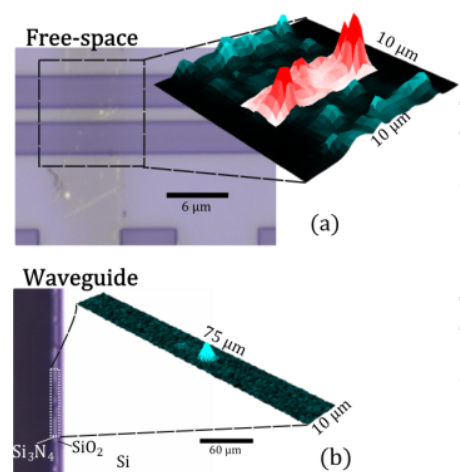

Raw Raman spectra

- NTP SERS through waveguide

... SiN background through waveguide

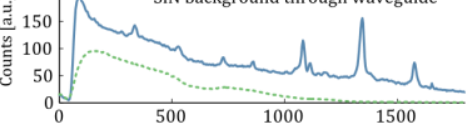

Stokes Shift [cm-1]

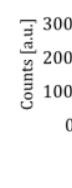

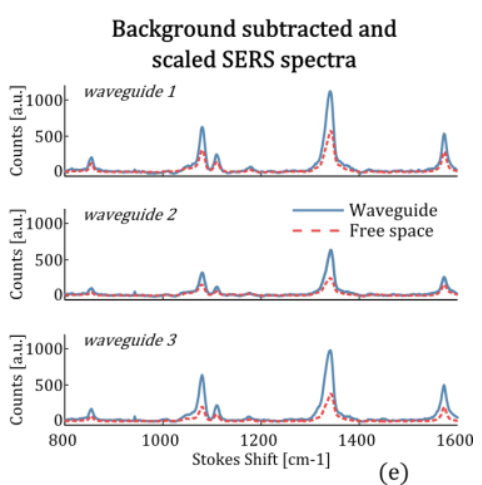

(d)

Figure 2 (a) Reflection image of a nanotriangle-patterned waveguide on which a high-resolution Raman scan is acquired in the area highlighted by the black rectangle. The inset in (a) shows an intensity profile of the $1337 \mathrm{~cm}^{-1}$ Raman peak of 4-NTP, with the nanotriangle-patterned waveguide highlighted in a red gradient. (b) Edge of the chip, scanned over the output facet of a waveguide proving that the 4-NTP spectra are originating from the waveguide. (c) Raw spectra collected through a reference waveguide (dashed green) and 4-NTP gold-coated waveguide (solid blue). (d) Comparison between waveguide (solid blue) and free-space measured SERS spectra (dashed red). The waveguide-coupled measurement is corrected for the $\mathrm{Si}_{3} \mathrm{~N}_{4}$ background. (e) Background subtracted and rescaled SERS spectra for a comparison between free-space and waveguide coupled measurements on three different waveguides, showing a $2.7 \pm 8$ stronger signal when collected through the waveguide.

monolayer (fig 1(b)). In order to quantify the SERS enhancement of these structures, the chip is labelled overnight with 4-nitrothiophenol (NTP, $1 \mathrm{mM}$ in ethanol), an aromatic compound that binds selectively to the gold through a gold-sulfur interaction. Finally, the chip is cleaved through the waveguides to enable end-fire coupling (fig 1(c)).

Surface-enhanced Raman spectra of gold-bound NTP molecules on identical waveguides are compared using both free-space (top-down) and waveguide-coupled excitation and collection under the same Raman microscope (WITec Alpha $300 \mathrm{R}+, \lambda_{\text {pump }}=785 \mathrm{~nm}$ ). In the former case, the microscope acquires a high resolution scan across the waveguide surface using a $250 \mathrm{~nm}$ step size, $0.2 \mathrm{~s}$ integration time and $500 \mu \mathrm{W}$ of pump power through a $100 \mathrm{x} / 0.9 \mathrm{NA}$ objective. Figure 2(a) shows that the signal exclusively originates from the $5 \mu \mathrm{m}$ wide gold-patterned strip. An average spectrum is calculated over 140 pixels spanning the patterned waveguide area. Variations along this area originate from the packing defects seen in fig 1(a). In the end-fire waveguide-coupled case, we align a $40 \mathrm{x} / 0.6$ objective to the cleaved $\mathrm{Si}_{3} \mathrm{~N}_{4}$ facet of the vertically positioned chip. Next, we acquire 140 spectra with a 0.2 $\mathrm{s}$ integration time and $250 \mu \mathrm{W}$ pump power. Fig 1(c) illustrates the coupling of the $785 \mathrm{~nm}$ pump light into the waveguide, where light scattering can be seen at the entrance facet, the nanotriangles section and the other end of the waveguide. This end is unused, as the Stokes scattered light is collected in back reflection through the same objective. However, the strong scattering at this point indicates that a considerable fraction of the pump light is not absorbed by the nanotriangles and hence this section can be extended to further increase Stokes conversion of the guided pump. Fig 2(b) shows a reflection image of the waveguide facet, where a scan along this facet proves that the Raman spectrum from the NTP molecules is only acquired when the light is coupled into the waveguide. The NTP SERS spectrum acquired from a nanotriangle-patterned waveguide contains also a strong contribution from the $\mathrm{Si}_{3} \mathrm{~N}_{4}$ waveguide core, measured from a reference waveguide and shown to the same scale in Fig 2(c). Fig 2(d) shows the raw acquired spectra in both excitation modes without the $\mathrm{Si}_{3} \mathrm{~N}_{4}$ background. After compensating for the coupling loss $(6.2 \pm 0.3 \mathrm{~dB})$ and differences in excitation power, as well as subtracting the remaining SERS background, the acquired NTP spectra on three different waveguides are a factor $2.7 \pm 0.8$ stronger when excited through the waveguide as compared to free-space (fig 2(e)).

This demonstration of using nanosphere lithography on $\mathrm{Si}_{3} \mathrm{~N}_{4}$ waveguides offers an easy method to functionalize integrated photonic circuits with nanoplasmonic antennas. We are working on further improvement of the signal enhancement by these kinds of patterns and on their integration with more complex photonic structures.

[1] S. Schlücker, "Surface-enhanced raman spectroscopy: Concepts and chemical applications," Angew. Chemie., 53, 4756-95, (2014).

[2] C. C. Evans, C. Liu, and J. Suntivich, "TiO 2 nanophotonic sensors for efficient integrated evanescent-Raman spectroscopy," ACS Photonics,

3, 1662-69, (2016).

[3] T. H. Stievater, S. A. Holmstrom, et al., "Trace-Gas Raman Spectroscopy Using Functionalized Waveguides," Optica, 3, 891-6, (2016).

[4] A. Dhakal, P. C. Wuytens, F. Peyskens, et al., "Nanophotonic Waveguide Enhanced Raman Spectroscopy of Biological Submonolayers," ACS Photonics, 3, 2141-49, (2016).

[5] A. Espinosa-Soria, A. Griol, and A. Martínez, "Experimental measurement of plasmonic nanostructures embedded in silicon waveguide gaps," Opt. Express, 24, 9592-601 (2016).

[6] F. Peyskens, A. Dhakal, P. Van Dorpe, et al., "Surface Enhanced Raman Spectroscopy Using a Single Mode Nanophotonic-Plasmonic Platform," ACS Photonics, 3, 102-108 (2016).

[7] A. Z. Subramanian, P. Neutens, A. Dhakal, et al., "Low-Loss Singlemode PECVD silicon nitride photonic wire waveguides for 532-900 nm wavelength window fabricated within a CMOS pilot line," IEEE Photonics J. 5, 2202809 (2013). 